\title{
EVALUACION CUALITATIVA Y CUANTITATIVA DE LA OFERTA \\ Y COSECHA DE BIOMASA HERBACEA POR ACROMYRMEX STRIATUS ROGER (HYMENOPTERA: FORMICIDAE) EN LA PROVINCIA DE LA PAMPA, ARGENTINA
}

\author{
QUALITATIVE AND QUANTITATIVE EVALUATION OF THE SUPPLY \\ AND HARVEST OF HERBACEOUS BIOMASS BY \\ ACROMYRMEX STRIATUS ROGER (HYMENOPTERA: \\ FORMICIDAE) IN LA PAMPA PROVINCE, ARGENTINA
}

\author{
Ana Armani ${ }^{1}$ \& Estela Quirán \\ ${ }^{1}$ Facultad de Ciencias Exactas y Naturales, Universidad Nacional de La Pampa; Av. Uruguay 151 L6300CLB, \\ Santa Rosa, La Pampa, Argentina. e-mail: emquiran@yahoo.com.ar
}

\begin{abstract}
In this paper we present a qualitative and quantitative evaluation of the supply and harvest of herbaceous biomass of the Acromyrmex striatus ant at a site in Santa Rosa city, La Pampa province, Argentina ( $\left.36^{\circ} 28^{\prime} \mathrm{S} 64^{\circ} 35^{\prime} \mathrm{W}\right)$. Annual input of green material to the nest was $0,14 \mathrm{~kg} /$ year.nest and $0,60 \mathrm{~kg} /$ (year.hectare); which is the lowest value reported for species of this genus. In the region studied, Acromyrmex striatus primarily cuts monocotyledonous, harvesting small amounts of all available plants. The presence of Acromyrmex striatus in this semiarid area would not decrease the availability of native grasses preferred by cattle.
\end{abstract}

KEYwords: Consumption, disponibility, vegetable patch, cutting ants, monocotyledonous, Argentina.

Las hormigas cortadoras (Hymenoptera: Formicidae) de la Región Neotropical son consideradas como equivalentes a los grandes herbívoros dominantes en otros continentes (Fowler et al. 1986; Fowler et al. 1991), debido a que colectan mucha más vegetación que cualquier otro grupo animal de diversidad taxonómica comparable (Hölldobler \& Wilson 1990). Las mismas pertenecen a la tribu Attini y comprenden dos géneros Atta y Acromyrmex, que se caracterizan por su amplia distribución y elevada densidad (DiehlFleig 1995).

Acromyrmex striatus Roger, comúnmente conocida como "hormiga cortadora colorada", es endémica de América del Sur. En Argentina se distribuye en la región árida y semiárida entre las provincias de
Jujuy (22 S) y Río Negro (42 S) (Kusnezov 1956; Bonetto 1959), donde el sol incide sobre el nido durante todo el año. Las colonias presentan actividad de forrajeo en los días cuyas temperaturas superan $\operatorname{los} 20^{\circ} \mathrm{C}$ y cortan vegetación rastrera y arbustiva baja, especialmente gramíneas (DiehlFleig 1995).

Existe una notoria falta de información sobre la composición de las dietas de las especies del género Acromyrmex en ambientes naturales (Della Lucía 1993; Pilati et al 1997), ya que la mayoría de los estudios sobre hormigas cortadoras han sido realizados en sistemas agrícolas tropicales más húmedos.

La presencia de nidos de estas hormigas podría comprometer la disponibilidad de ciertas especies 
vegetales naturales, necesarias para la alimentación de las especies herbívoras de la región y del ganado doméstico.

El objetivo de esta contribución es realizar una evaluación cualitativa y cuantitativa de la oferta y del material acarreado por Acromyrmex striatus hacia los nidos.

El trabajo se realizó en el campo de la Facultad de Agronomía de la Universidad Nacional de La Pampa $\left(36^{\circ} 28^{\prime}, \mathrm{S} 64^{\circ} 35^{\prime}, \mathrm{W}\right)$ sito en el Departamento Capital, de la provincia de La Pampa (Argentina). Los muestreos de cosecha de las hormigas y de oferta vegetal se realizaron en los meses de octubre y diciembre de 2005 y abril de 2006. La duración de los mismos fue de ocho días en cada oportunidad y sobre cuatro colonias elegidas al azar, en cada ocasión. El área de influencia de los nidos, de forma circular, fue establecida considerando un radio de 30 metros alrededor de la entrada principal, en todos los meses en los que se realizaron los muestreos. La oferta de fitomasa herbácea se determinó por cortes al ras de 35 muestras tomadas con cuadrantes de $0,25 \mathrm{~m}^{2}$ distribuidos al azar en el área de influencia de cada uno de los hormigueros seleccionados. También se colectó la broza. La totalidad del material verde fue identificado, secado en estufa durante 3 días a $65^{\circ} \mathrm{C}$ y pesado. Para conocer la diversidad del estrato graminoso - herbáceo en términos de fitomasa vegetal se calculó el Indice de Diversidad de Simpson (1949):

$$
\mathrm{ID}=1 / £(n i / N)^{2}
$$

siendo, $n i$ : la biomasa de la especie herbácea " $i$ " y $N$ el total de biomasa herbácea.

Para estimar la cantidad cosechada por A. striatus se recolectó durante 6 minutos cada 2 horas el total del material que las hormigas acarreaban a sus nidos. El mismo fue luego secado, pesado e identificado (cuando el tamaño lo permitía) en el laboratorio. La cosecha ( $\mathrm{kg} /$ año.hormiguero) se estimó multiplicando el promedio de los consumos diarios de los ocho muestreos de octubre, diciembre y abril, respectivamente, por los días de actividad de las hormigas, aquellos con temperaturas superiores a $20^{\circ} \mathrm{C}(\mathrm{n}=236)$, durante el año del estudio. Un censo de hormigueros activos en cada muestreo permitió cuantificar la cosecha realizada por las hormigas.
La oferta vegetal de herbáceas en pie y broza mostró variaciones según el momento del año (Tabla I). Se observa que a partir de octubre la vegetación aérea (ID: 2,31), compuesta de monocotiledóneas y dicotiledóneas, aumenta hasta alcanzar la máxima disponibilidad en diciembre (ID: 4,21) con predominio de monocotiledóneas; disminuyendo hacia abril (ID: 2,71). En octubre la mayor proporción en la oferta vegetal correspondió a Panicum bergii Arech, Crepis foetida L. y Lolium multiflorum Lam.; mientras que en diciembre se destacan Cynodon dactylon (L.), Eleusine tristachya (Lam.), Hypochoeris pampasica Cabrera, Eragrostis lugens Ness, Bromus brevis Ness y Solanum eleagnifolium Carles; por último, en abril las especies más abundantes fueron Eragrostis lugens Ness, Cynodon dactylon (L) yL. multiflorum Lam. (Tabla I). De estas especies, Lolium multiflorum es considerada valiosa, para el manejo del ganado doméstico de la zona (Nazar Anchorena 1988).

El material forrajeado por A. striatus en cada momento del muestreo, se componía de fragmentos de hojas, tallos y flores, en su mayoría pertenecientes a las especies vegetales mencionadas anteriormente. El presente estudio podría complementarse con la cuantificación de dichos materiales. El forrajeo diario promedio fue de $0,62 \mathrm{gr} /$ hormiguero.día y la densidad media de hormigueros activos en el área de estudio fue de 4,32 hormigueros/ha. El acarreo promedio de $A$. striatus fue de $0,14 \mathrm{~kg} /$ año.hormiguero y de $0,60 \mathrm{~kg}$. año/ha. Con estos resultados se podría inferir que $A$. striatus es una especie generalista que utiliza los recursos vegetales que el ambiente le ofrece y en pequeñas cantidades, que no afectarían a las demás especies del sistema. El forrajeo anual obtenido para A. striatus de 0,14 $\mathrm{kg} / \mathrm{año}$.hormiguero es bajo comparado con el de Acromyrmex octospinosus Reich que es de 0,94 kg/ año.hormiguero y Acromyrmex landolti Forel de 0,42 - 2,2 kg/año.hormiguero (Fowler et al. 1991). Estas últimas especies se distribuyen en climas subtropicales a tropicales, más húmedos, con abundante disponibilidad de vegetación. El estudio de A. striatus se realizó en una región de clima de transición de subtemplado a semiárido, donde la oferta vegetal es menor. A su vez, el acarreo de material al nido de A. striatus se diferencia ampliamente del de Acromyrmex lobicornis Emery ("hormiga cortadora negra") de la región semiárida 
Evaluación de la cosecha de A. striatus Roger. Armani, A. \& E. Quirán

de La Pampa, Argentina. Esta última especie cosecha $8,47 \mathrm{Kg}$ /año.hormiguero (Pilati et al. 1997). Esta gran diferencia podría deberse a que esta especie es capaz de cosechar bajo condiciones ambientales muy variables. Además, presenta una mayor densidad de nidos en el área de distribución (Quirán \& Pilati, 1997); éstos son más grandes (Quirán \& Pilati, 1998) y populosos, que los de $A$. striatus, por lo que demandarían una mayor cosecha. Además, A. lobicornis corta con temperaturas inferiores a $23^{\circ} \mathrm{C}$-por ello durante el verano su máxima actividad en la región semiárida de La Pampa se da durante la noche (Pilati et al 1997)- o sea que su patrón de cosecha comprende de 14 a 16 hs. por día durante gran parte del año (Pilati \& Quirán 1996). Acromyrmex striatus presenta una baja densidad de hormigueros en el terreno, con menos cantidad de individuos (Diehl-Fleig 1995). Es una especie que sólo forrajea a temperaturas superiores a $20^{\circ} \mathrm{C}$ (DiehlFleig 1995), por lo que su actividad de cosecha es muy breve, disponiendo solo durante los meses de verano de varias horas por día con esa temperatura. Tomados en su conjunto, los resultados del presente trabajo indican que A. striatus es una especie generalista que cosecha las monocotiledóneas disponibles en el ambiente y en cantidades poco importantes, por lo que no comprometería el recurso vegetal utilizable por el ganado doméstico.

TABLa I. Variación estacional de la oferta de vegetales verdes y broza en gr $/ \mathrm{m}^{2}$ e Indice de Diversidad de Simpson.

TABLE I: Seasonal variation of the supply of green vegetables and rubbish in $\mathrm{gr} / \mathrm{m}^{2}$ and Index of Diversity of Simpson.

\begin{tabular}{lccc}
\hline VEGETALES VERDES & OCTUBRE & DICIEMBRE & ABRIL \\
\hline Bothriochloa springfieldii & 0 & 0,84 & 10,68 \\
Bromus brevis & 1,36 & 30,59 & 0 \\
Coniza bonariensis & 2,1 & 0 & 0 \\
Crepis foetida & 66,15 & 0 & 0 \\
Cynodon dactylon & 6,88 & 259,91 & 111,43 \\
Descurainia argentina & 0 & 1,5 & 0 \\
Eleusine tristachya & 0 & 182,84 & 1,44 \\
Eragrostis lugens & 6,84 & 31,55 & 152,78 \\
Eustachys retusa & 2,01 & 17,72 & 0 \\
Gamochaeta pensylvanica & 0 & 11,35 & 0 \\
Graphalium gaudichaudianum & 0 & 10,6 & 0 \\
Hypochoeris pampasica & 0 & 110,51 & 0 \\
Lactuca serriola & 0 & 0,4 & 0 \\
Lepidium bonaeriensis & 0 & 0,3 & 0 \\
Lolium multiflorum & 36,9 & 0 & 30,32 \\
Medicago lupulina & 0 & 1,2 & 0 \\
Panicum bergii & 200,47 & 9,6 & 0 \\
Petrorhagia nanteuillii & 3,42 & 0 & 0 \\
Phacelis retusa & 0 & 2,13 & 0 \\
Sanchus oleraceus & 0 & 0,08 & 0 \\
Setaria parviflora & 0 & 0 & 0,48 \\
Solanum eleagnifolium & 0 & 29,31 & 8,67 \\
Veronica arvensis & 0 & 0,16 & 0 \\
VEGETALES vERDES & 326,13 & 700,59 & 315,8 \\
BROZA & 401,26 & 211,19 & 458 \\
ID DE SIMPsON & 2,31 & 4,21 & 2,71 \\
\hline
\end{tabular}




\section{AGRADECIMIENTOS}

Al Ing. O. Martínez por la identificación de la vegetación y a la Facultad de Ciencias Exactas y Naturales (UNLPam) por el apoyo financiero. Al Dr. Lohengrin Cavieres y un revisor anónimo que realizaron valiosos aportes sobre una versión previa del trabajo.

\section{BIBLIOGRAFIA}

Bonetto, A.A. 1959. Las hormigas "cortadoras" de la Provincia de Santa Fe (Gén. Atta y Acromyrmex). Minist. de Agric. y Ganadería de Santa Fe. Dir. Recursos Naturales. Santa Fe. Argentina. 89 pp.

Della Lucia, T.M.C. 1993. As Formigas Cortadeiras. Ed. Terezinha M.C.Della Lucía, Viçosa.

DieHl-Fleig, E. 1995. Formigas: organizaçao social e ecologia comportamental. São Leopoldo: Ed. Unisinos. $168 \mathrm{pp}$.

Fowler, H. G., L. C. Forti, V. Pereira-Da-Silva \& N. B. SAES. 1986. Economics of grass-cutting ants. En: Fire ants and leaf-cutting ants: biology and management. (Eds. Lofgren, C. S. \& R. K. Vander Meer), pp. 18-35. Boulder, Westview Press. E.E.U.U.

Fowler, H. G.; L. C. Forti; C. R. F. Brandao; J. C. Delabie \& H. L. VASCONCELOS. 1991. Ecología nutricional de formigas. En: Ecología nutricional de insectos
(Eds. Panizzi, A. P. \& J. R. P. Parra). pp. 131223. São Paulo, Manole.

Hölldobler, B. \& E.O. Wilson. 1990. The Ants. Cambridge, Harvard University Press. Pp. 732.

Kusnezov, N. 1956. Claves para la identificación de las hormigas de la fauna argentina. IDIA $\mathrm{N}^{\circ} 100$ : 1 56.

NazarAnchorena, J. B. 1988. Pastizales Naturales de La Pampa. 2. Manejo de los mismos. Convenio AACREA - Prov. de La Pampa. 112 pp.

Pilati, A. \& E. Quirán, 1996: Patrones de cosecha de Acromyrmex lobicornis (Formicidae:Attini) en un pastizal del Parque Nacional Lihué Calel, La Pampa, Argentina. Rev. Ecología Austral 6:123126.

Pilati, A., E. M. Quirán \& H. D. Estelrich. 1997. Actividad forrajera de Acromyrmex lobicornis Emery (Hymenoptera: Formicidae) en un pastizal natural semiárido de la provincia de La Pampa (Argentina). Ecología Austral 7: 49-56.

Quirán, E. \& A. Pilati. 1997: Distribución espacial de los hormigueros de Acromyrmex lobicornis (Emery, 1887) en un sitio natural semiárido de la Provincia de La Pampa. Rev. de la Soc. Entomol. Argent. 56(1-4):155-157.

Quirán, E. \& A. Pilati. 1998. Estructura de los hormigueros de Acromyrmex lobicornis (Hymenoptera:Formicidae) en un sitio natural semiárido de La Pampa, Argentina. Rev. de la Soc. Entomol. Argent. 57(1-4):45-48.

Simpson, E. H. 1949. Measurement of diversity. Nature 163: 688 . 